\title{
THE INFLUENCE OF A VASCULAR FACTOR ON THE DISTRIBUTION OF SYMMETRICAL CEREBRAL CALCIFICATIONS
}

BY

\author{
R. M. NORMAN and H. URICH \\ From the Burden Neuropathological Laboratory, Frenchay Hospital, Bristol
}

Calcification of cerebral capillaries and smaller blood vessels appears to be a response on the part of the tissues to a wide range of pathological influences. Various forms of encephalitis and endocrine anomalies, particularly hypoparathyroidism, together account for the greatest number of cases to which a definite aetiology may be assigned, while familial instances, often associated with oligophrenia or microcephaly, are well known. Irrespective of aetiology and in the large group of cryptogenic cases, the localization of the calcifications is singularly uniform. The putamina and central parts of the cerebellar hemispheres usually show dense deposits which by confluence often form "brain stones" of massive size. The globus pallidus, thalamus, parts of the cerebral cortex and centrum semiovale are also bilaterally implicated though the complete pattern is not always present.

These favoured sites for calcification have been recognized for many years but little attention has been paid to their finer topographical features. In the present case we have been struck by the fact that the site of the calcifications is remarkably similar to that of ischaemic lesions often found in cases of birth injury or associated with circulatory disturbances in later life. This is particularly apparent in the involvement of the deeper parts of the gyral walls and of the boundary zones between major cortical arterial territories. Moreover, the distribution of the calcifications in the basal ganglia has much in common with that of status marmoratus. Our purpose in this paper is to develop this comparison more fully, for we believe that the exposure of a vascular factor sheds light on the pathogenesis of cerebral calcification.

\section{Case Report}

Clinical History.-J.P., a man, died at the age of 49 years. His parents were dead at the time he came under supervision and nothing is known about the family history or his early childhood except that he suffered from epilepsy and had been admitted to a hospital at the age of 3 years for "paralysis of the legs".
From the age of 5 years, however, he was able to attend an ordinary elementary school but at 11 years he was transferred to a special school. On leaving he worked as an errand boy for four years and then in a factory for two years until discharged for incompetence. When 28 years old he was certified as a feeble-minded person. A year before his death he was transferred to Stoke Park Hospital for mental defectives. His mental age as estimated by the Terman-Merrill scale was then only 4 years 8 months (I.Q. 30). He had preserved traces of verbal ability well above his apparent level at the time of testing and in the psychologist's opinion he was an imbecile who had deteriorated considerably. The impression was given of psychosis superimposed on mental defect. No abnormality was found on neurological examination. The blood Wassermann reaction was negative. During the last few months of life, however, there ensued a rapid physical and mental deterioration. $\mathrm{He}$ developed increasing flexion rigidity of the arms and extension rigidity of the legs. Five months before death the fingers of the left hand became painful and the hand and forearm became swollen. $X$-ray examination showed bone absorption of the distal ends of the third and fourth proximal phalanges and this was regarded as due to a chronic infective condition. The serum calcium was $7.2 \mathrm{mg} . \%$, serum potassium $21.2 \mathrm{mg} . \%$, alkaline phosphatase 14.7 King-Armstrong units, plasma cholesterol $100 \mathrm{mg} . \%$, and blood urea $38 \mathrm{mg} . \%$. In the final stages of his illness the patient became apathetic, bedridden, and incontinent, death occurring from bronchopneumonia. Three months before death he had the only grand mal seizure recorded during his long period in hospital. Tetany had never been noted.

\section{Examination of the Central Nervous System}

Macroscopic.-The fixed brain weighed 1,398 g., of which the cerebellum and brain-stem accounted for $142 \mathrm{~g}$. The leptomeninges, vessels, and convolutional pattern were normal. On dissection marked calcification of the tissues was found in both putamina where several small "brain stones" were easily detachable from the tissues. Patchy grittiness was also noted in the region of the dentate nuclei, in the central white matter, and in the depths of gyral walls, particularly at the frontal poles and in the second frontal gyri. 


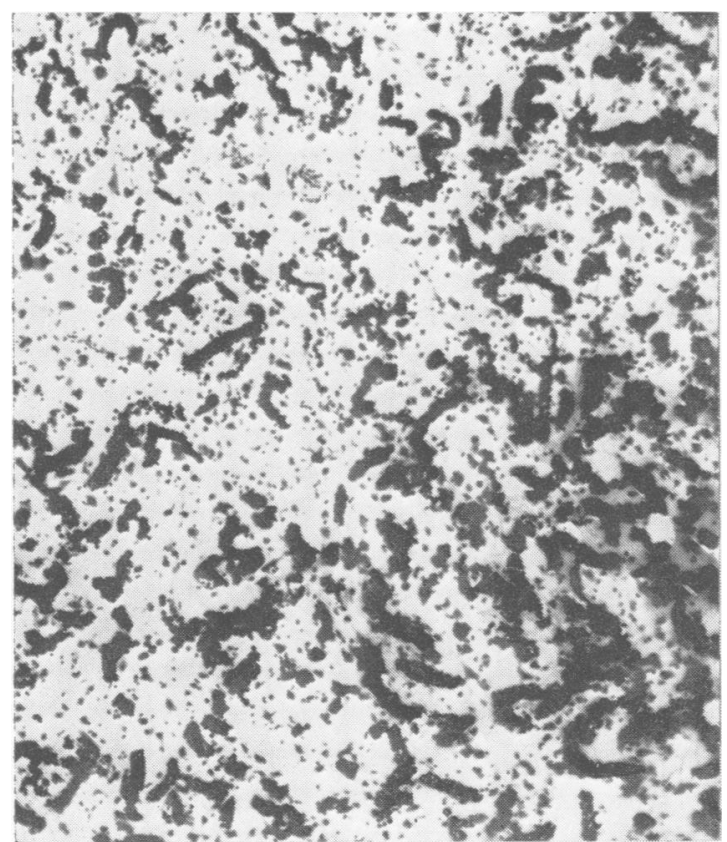

FIG. 1.-Incrustation of cortical capillaries in the second frontal convolution. Carbol azura $\times 90$.
Microscopic.-Large celloidin survey sections, mostly of the complete hemispheres, were stained for nerve cells and myelin. The Holzer stain for fibrous neuroglia was used on de-celloidinized material. Similar methods were used with representative sections from the cerebellum, brain-stem, and spinal cord.

With a few exceptions to be noted later the lesions found in this brain consisted in calcification of the smaller intracerebral blood vessels which took the form of pericapillary droplets often coalescing into a sheath-like investiture of the vessel wall (Fig. 1). In the more densely calcified areas, especially in the putamen, large numbers of palely-staining calcospherites were present among the encrusted capillaries. All concrements stained bright red with the periodic-acid-Schiff method and a pale blue with Alcian blue.

Cerebral Cortex.-Pericapillary calcification was found only in the walls of gyri and floors of sulci where the deeper layers were mainly involved. Large numbers of nerve cells remained between the calcified vessels and there was no evidence of atrophy, gliosis, or neuronal incrustation. In the frontal lobes the vessels in the basal parts of the second frontal gyri were calcified (Figs. 2 and 3). On

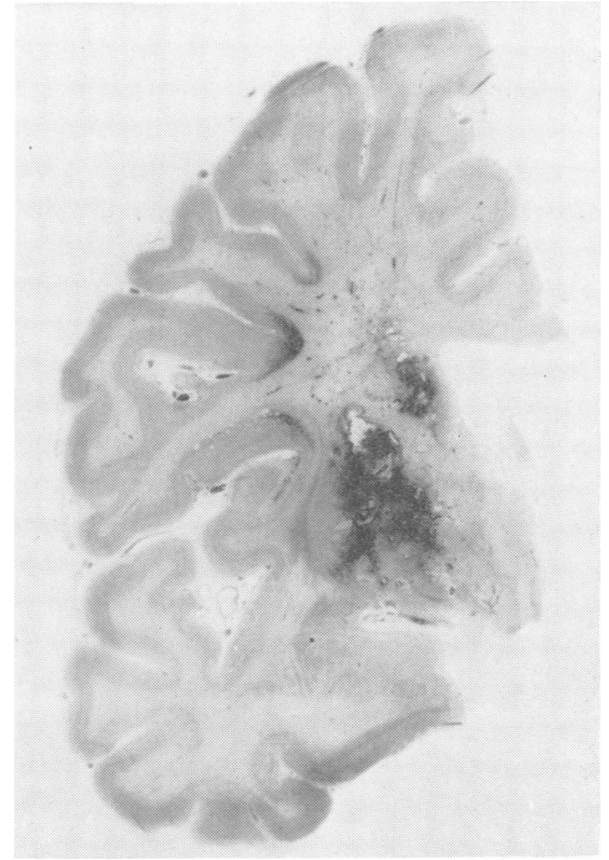

FIG. 2.-Left cerebral hemisphere: calcium deposits are prominent at the base of the second frontal convolution, beneath the superior limiting sulcus of the insula, in the caudate nucleus, putamen, and external segment of the pallidum. Carbol azure $\times 1$.

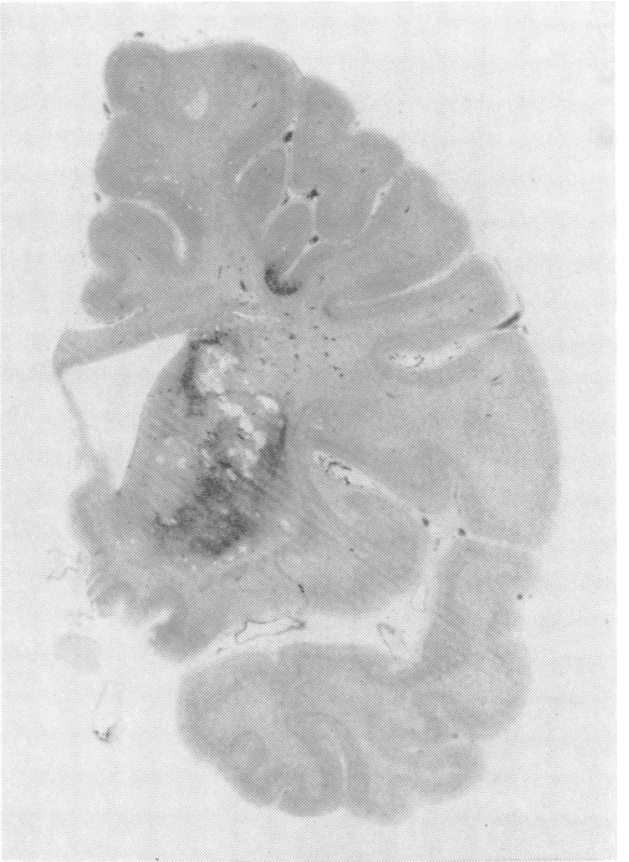

FIG. 3.-Right cerebral hemisphere showing similar distribution of lesions at the level of the head of the caudate nucleus. The holes are due to large concrements falling out. Carbol azure $\times 1$. 
the orbital surface the grey matter bounding the depths of the orbital sulcus was symmetrically affected in this way and near the frontal pole a prominent patch of calcification was also present in the medial part of the frontal lobe of both sides (Fig. 4). The cortex of the insula was normal except for calcifications surrounding the superior limiting sulcus, again an approximately symmetrical feature (Figs. 2 and 7).

In the temporal lobes capillary incrustations selectively involved the basal parts of the middle temporal gyrus. The hippocampal formations were not affected.

The parietal and occipital lobes were intact except for a patch of dense calcification in the depth of the left calcarine area. Here the laminar distribution of the lesion was striking, the granular layer being almost solely involved and mainly in its deeper parts (Fig. 5).

Cerebral White Matter.-Capillary incrustations were rare, the deposits being found in the adventitia or media of larger vessels, especially the veins. All transitions from isolated droplets to complete encirclement or total obliteration of the vessels were

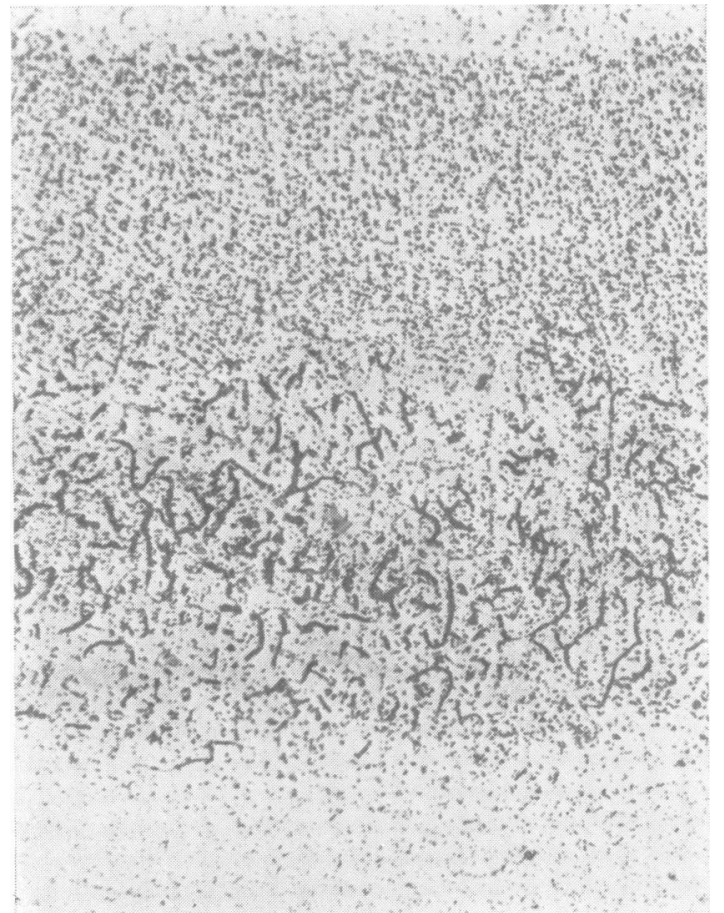

Fig. 5.-Left occipital cortex: the incrustation of capillaries has a laminar distribution involving the deeper parts of the granular layer. Carbol azure $\times 60$.

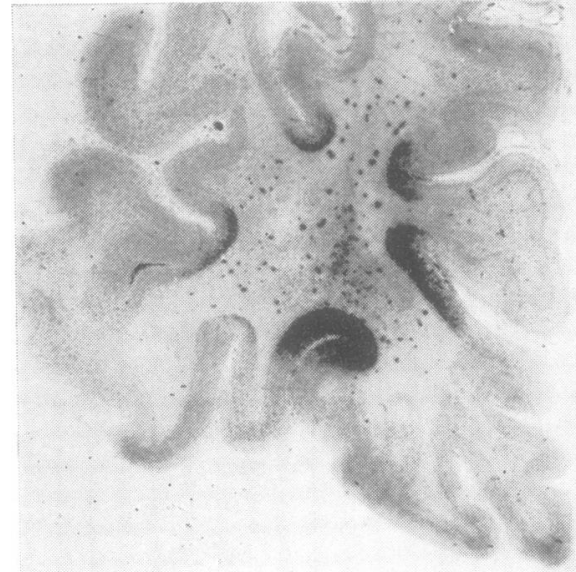

Fig. 4.-Right frontal cortex near pole: calcifications are present in, but not confined to, the arterial boundary zones of the convexity and orbital surface. Carbol azure $\times 1.6$.

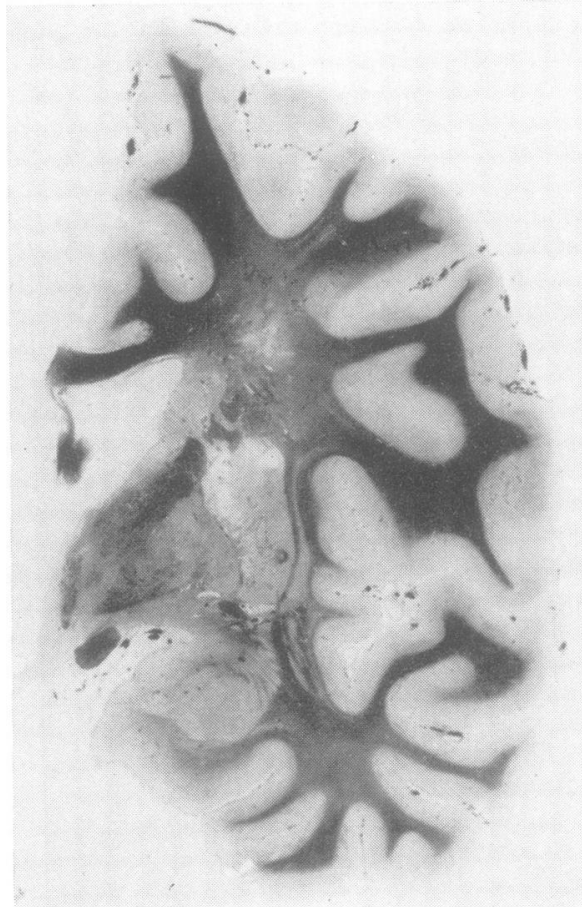

FIG. 6.-Right cerebral hemisphere: an ill-defined area of demyelination is present in the centrum semiovale. Heidenhain's myelin stain $\times 1$. 


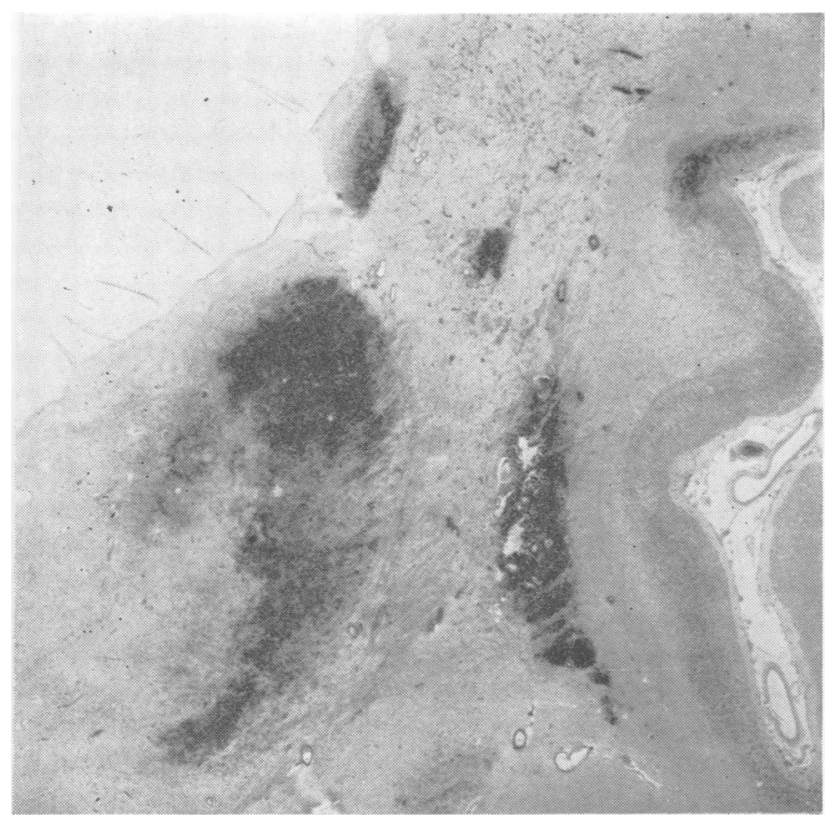

FIG. 7

FIG. 7.-Right thalamus: the main mass of calcifications occupies the ventrolateral nucleus; deposits are also present in the putamen, caudate nucleus, and upper part of the insular cortex. Carbol azure $\times 3 \cdot 5$.

Fig. 8.-Cerebellum: calcium deposits can be seen in the dentate nucleus, the central white matter, and the granular layer of the deeper parts of the folia. von Kossa's method $\times 3 \cdot 3$.

seen. In some vessels a broad acellular zone, amorphous or fibrillary in character, was interposed between the endothelial layer and the outer ring of calcium. An ill-defined, diffuse loss of myelinated fibres, shading off towards the periphery, was present in the centrum semiovale (Fig. 6). Fibrous gliosis was observed in the neighbourhood of heavily calcified vessels.

Basal Ganglia.-The putamina were the most heavily calcified areas in the brain, the process affecting mainly the capillaries but also the larger vessels. Calcospherites and larger, palely staining conglomerates were common in the central parts of the nucleus where the parenchyma was almost completely replaced by calcium or neuroglia. At some levels a narrow lateral or basal strip of normal tissue was preserved. The head and body of the caudate nucleus on each side showed dense pericapillary calcifications confined to the lateral part adjoining the internal capsule. The external segment of the globus pallidus was also severely affected while in the internal segment the larger vessels rather than the capillaries were calcified and

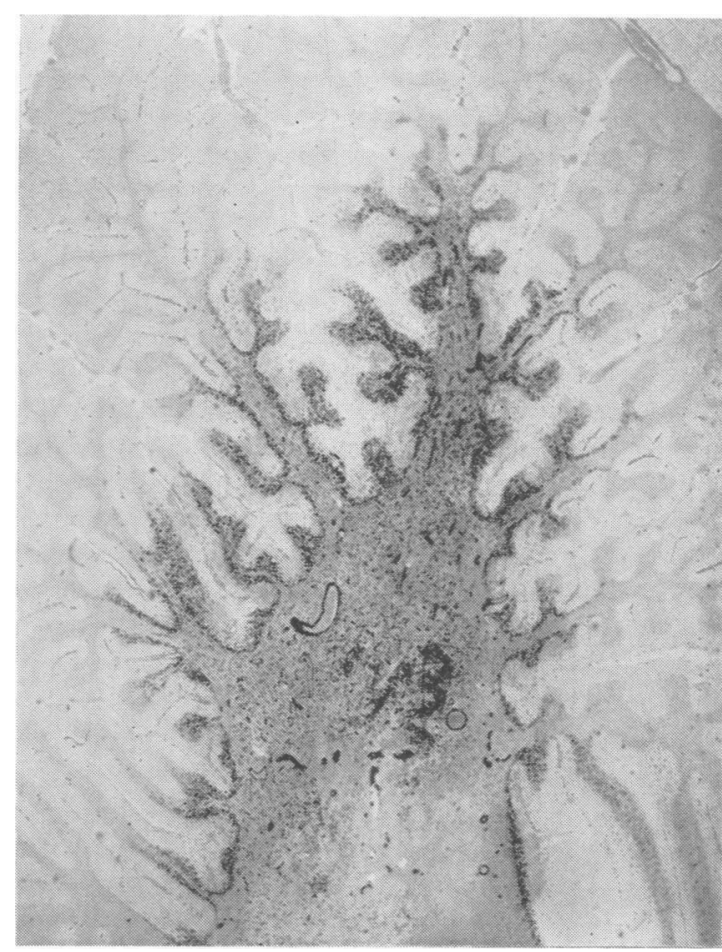

FIG. 8

numerous free concretions were present (Figs. 2, 3 , and 7 ). The amygdaloid nuclei were normal. In the posterior half of the thalami the lateral nuclei were conspicuously involved (Fig. 7), but numerous nerve cells were preserved amid the calcified vessels. The medial nuclei were less affected. Only the uncalcified centromedian nuclei were atrophic, evidently as a consequence of the striatal damage.

The subthalamic and red nuclei were patchily calcified.

Cerebellum.-Dense pericapillary incrustations were seen in the dentate nuclei, particularly in the dorsal and posterior parts. The white matter lying subjacent to the semilunar lobules and the adjoining quadrangular lobe, including the deeper parts of the gyral cores, was also the site of calcification of both large and small vessels. In this region the loss of myelin was pronounced. The capillaries of the granular layer of the deeper folia of the semilunar lobules were conspicuously affected. This distribution of the calcifications was well demonstrated in von Kossa preparations (Fig. 8). Purkinje cells were not lost or calcified. 
In the brain-stem the only lesions seen were slight losses of nerve cells in the nucleus pontis and inferior olives. The spinal cord, ependyma, choroid plexus, and meninges were unremarkable.

Chemical analysis of a large concrement removed from the putamen showed an enormous preponderance of calcium over iron (calcium $16,800 \mathrm{mg} . \%$, iron $25.5 \mathrm{mg}$. $\%$ wet basis).

\section{Discussion}

Cerebral calcification had not been suspected in this patient during life and thus no appropriate radiological or biochemical investigations had been carried out. It was, however, fortunate that on one occasion the serum calcium had been estimated and found to be reduced, since in the light of the subsequent findings it seemed probable that parathyroid deficiency had been present. Hypoparathyroidism appears to be the most frequent aetiological factor in symmetrical cerebral calcification of the strio-dentate type, the association having been found in $28 \%$ of cases of idiopathic hypoparathyroidism and in $48 \%$ of cases of Albright's pseudohypoparathyroidism (Bronsky, Kushner, Dubin, and Snapper, 1958). The condition is often referred to as "Fahr's disease" (Fahr, 1930), but since many examples of symmetrical cerebral calcification have been well described from the time of Mallory (1896) onwards the use of this eponym has little justification apart from its brevity.

Chemical and histochemical analysis of the concrements have shown two components. Calcium, not iron, is present in large amounts (Wachsmuth and Löwenthal, 1952). An organic matrix is also invariably present, the "pseudo calcium" of Spatz (1922), recently renamed "neurogel" by Bochnik (1950). This matrix, generally regarded as proteinous by early writers on the subject, has the staining reactions of an acid mucopolysaccharide. It has often been assumed that the formation of matrix precedes calcification, but this seems improbable in the light of Gedigk and Strauss' (1954) demonstration that histiocytes are capable of producing a mucopolysaccharide matrix in response to the deposition of mineral salts.

The important observation was made in Beyme's (1945-46) case that calcium is present in the concrements in the form of hydroxyl apatite or "bone salt". It thus became evident that the conjunction of matrix and calcium salts was not a simple one but involved a highly complex process of synthesis, presumably by mesenchymal cells, as in bone formation. Attention was diverted from the problem of altered capillary permeability and Beyme suggested that the vessels acted merely as con- venient surfaces for the precipitation of tissue colloids. We believe that this extreme viewpoint should be modified so as to take into account the characteristic localization of the calcifications within the brain, for this regional selectivity bears witness to a vascular factor of importance.

The presence of calcifications in cortical boundary zones lying between major arterial territories cannot be dismissed as fortuitous, since ischaemic lesions of similar localization have been found in a variety of arterial diseases and in birth injury (Meyer, 1953; Norman, Urich, and McMenemey, 1957). In our present case the basal parts of the second frontal gyri were widely affected and on each side the calcifications implicated the orbital part of the frontal lobe where they lay between the fields of the anterior and middle cerebral arteries. In the temporal lobes the boundary zone between the middle and posterior cerebral arteries was also selectively involved. Calcification of the second frontal gyrus has been previously noted in other cases of symmetrical cerebral calcification (Beyme, 1945-46; Chavany, van Bogaert, and Houdart, 1949), but its significance has not been discussed. Even in more widely calcified brains, as in that illustrated by Erbslöh and Bochnik (1958), this site of predilection has shown an evident accentuation of the deposits. In a familial example of this disorder at present under examination in this laboratory we have also seen calcifications occupying the parieto-occipital boundary zones. Finally, in the cerebellum of the present case the preponderance of calcifications in the neighbourhood of the horizontal fissure is suggestive of an analogous lesion in the terminal distributions of the superior and inferior cerebellar arteries.

Calcification mainly confined to parts of the fourth layer of the calcarine cortex was also present in our case. A similar pericapillary distribution of mineral deposits has previously been described in general paresis by Löwenberg (1928) and we have seen it in our own material in Sturge-Weber's disease and in post-encephalitic brain damage. The same layer is also frequently picked out by the perivascular plaques described by Morel and Wildi (1955) in certain cases of senile dementia.

There are also striking similarities between the localization of the calcifications of the basal ganglia and the lesions of status marmoratus following birth injury. In the latter condition the preferential sites for marbling, nerve cell loss, and gliosis are the putamen, the outer segment of the pallidum, the ventro-lateral portion of the head of the caudate nucleus, and the lateral nucleus of the thalamus (Norman, 1958).

From these comparisons certain deductions 
appear to follow. It is evident that symmetrical cerebral calcification tends to occupy preferential sites, irrespective of the aetiological factors concerned. That this distribution may be determined by regional peculiarities of blood vessels is suggested by the finding of calcified capillaries occupying the boundary zones between major arterial territories. Boundary zone lesions have hitherto been regarded as a characteristic consequence of a diminished arterial blood flow, whether due to obliterative disease or a fall in systemic pressure. In the type of cerebral calcification under consideration the large arteries are not narrowed by mineral deposits or degenerative changes and it would seem that the capillaries of the boundary zones are primarily affected. The theory that regional vascular peculiarities are responsible for the localization of the calcifying lesions derives further support from the laminar distribution of the calcifications in the visual cortex and from the topography of the lesions in the basal ganglia. It is generally admitted that vascular factors play a part in the pathogenesis of status marmoratus and the occurrence of lesions of almost identical distribution in an otherwise dissimilar condition suggests the presence of a local factor common to both.

This vascular factor is doubtless only one link in the chain of complex reactions underlying the process of cerebral calcification and it is tempting to equate it with an enhanced capillary permeability. Löwenthal (1948), who was among the first to recognize the association of hypoparathyroidism with symmetrical cerebral calcification, concluded that one feature of the deranged metabolism might be the facilitated passage of calcium salts into the perivascular tissues. Some experimental support has been given to this theory by the work of Stern, Belkina, and Zlatowierow (1928) who demonstrated changes in the blood-brain barrier in conditions of parathyroid deficiency. Altered capillary permeability may thus provide a link between the two main aetiological factors which have been established in states of cerebral calcification, namely, endocrine disturbances and infections of the nervous system.

\section{Summary}

A man aged 49 years at death had shown signs of slowly progressive mental deterioration since the age of 11. During the last few months of life this was accompanied by increasing muscular rigidity. Examination of the brain showed calcification of the capillaries and smaller blood vessels bilaterally in the corpus striatum, thalamus, the region of the dentate nucleus, and in parts of the cerebral cortex. In the cerebral cortex only the deeper parts of certain gyri were affected and there was a strikingly symmetrical involvement of the boundary zones between fields of supply of major arteries. This distribution of the calcified vessels closely resembles that of ischaemic lesions sometimes found as a consequence of cerebral circulatory disturbances caused by birth injury, cardiac arrest, or arterial disease. It is considered that a vascular factor, possibly a peculiarity of capillary permeability, may be responsible for the localization of the lesions both in symmetrical cerebral calcifications and in these otherwise unrelated conditions.

We are greatly indebted to Mr. A. H. Tingey for his chemical findings and to Dr. W. A. Heaton-Ward for the use of the clinical notes in this case. This work was carried out with the help of the Nuffield Foundation.

\section{REFERENCES}

Beyme, F. (1945-46). Schweiz. Arch. Neurol., 56, 161 and 57, 16. Bochnik, H. J. (1950). Arch. Psychiat. Nervenkr., 184, 201.

Bronsky, D., Kushner, D. S., Dubin, A., and Snapper, I. (1958). Medicine (Baltimore), 37, 317.

Chavany, J. A., Bogaert, L. van, and Houdart, R. (1949). Mschr. Psychiat. Neurol., 117, 77.

Erbslöh, F., and Bochnik, H.'(1958). In Henke-Lubarsch' Handbuch der speziellen pathologischen Anatomie, Vol. 13, Pt. IIB, p. 1769. Springer, Berlin.

Fahr, T. (1930). Zbl. allg. Path., 50, 129.

Gedigk, P., and Strauss, G. (1954). Virchows arch. path. Anat., 326, 172 .

Löwenberg, K. (1928). J. Psychol. Neurol., 36, 81.

Löwenthal, A. (1948). Acta neurol. psychiat. belg., 48, 613.

Mallory, F. B. (1896). J. Path. Bact., 3, 110.

Meyer, J. E. (1953). Arch. Psychiat. Nervenkr., 190, 328.

Morel, F., and Wildi, E. (1955). Schweiz. Arch. Neurol., 76, 174.

Norman, R. M. (1958). In Temporal Lobe Epilepsy, p. 203, ed. Maitland Baldwin and Pearce Bailey. Charles C. Thomas, Springfield, Illinois.

_- Urich, H., and McMenemey, W. H. (1957). Brain, 80, 49.

Spatz, H. (1922). Z. ges. Neurol. Psychiat., 77, 261.

Stern, L., Belkina, L. G., and Zlatowierow, A. O. (1928). C. R. Soc Biol. (Paris), 99, 536.

Wachsmuth, N., and Löwenthal, A. (1952). In Trav. Inst. Bunge, 7. Reprinted from Acta neurol. psychiat. belg., 50, 305 (1950). 